\title{
Probe of the Anomalous Quartic Couplings with Beam Polarization at the CLIC
}

\author{
A. Senol, ${ }^{1}$ M. Köksal, ${ }^{2}$ and S. C. İnan ${ }^{3}$ \\ ${ }^{1}$ Department of Physics, Abant Izzet Baysal University, 14280 Bolu, Turkey \\ ${ }^{2}$ Department of Optical Engineering, Cumhuriyet University, 58140 Sivas, Turkey \\ ${ }^{3}$ Department of Physics, Cumhuriyet University, 58140 Sivas, Turkey
}

Correspondence should be addressed to S. C. İnan; sceminan@cumhuriyet.edu.tr

Received 9 September 2016; Revised 30 November 2016; Accepted 21 December 2016; Published 11 January 2017

Academic Editor: Edward Sarkisyan-Grinbaum

Copyright (C) 2017 A. Senol et al. This is an open access article distributed under the Creative Commons Attribution License, which permits unrestricted use, distribution, and reproduction in any medium, provided the original work is properly cited. The publication of this article was funded by SCOAP $^{3}$.

\begin{abstract}
We have investigated the anomalous quartic couplings defined by the dimension- 8 operators in semileptonic decay channel of the $e^{+} e^{-} \rightarrow v_{e} W^{-} W^{+} \bar{v}_{e}$ process for unpolarized and polarized electron (positron) beam at the Compact Linear Collider. We give the $95 \%$ confidence level bounds on the anomalous $f_{S 0} / \Lambda^{4}, f_{S 1} / \Lambda^{4}$, and $f_{T 0} / \Lambda^{4}$ couplings for various values of the integrated luminosities and center-of-mass energies. The best sensitivities obtained on anomalous $f_{S 0} / \Lambda^{4}, f_{S 1} / \Lambda^{4}$, and $f_{T 0} / \Lambda^{4}$ couplings through the process $e^{+} e^{-} \rightarrow v_{e} W^{-} W^{+} \bar{\nu}_{e}$ with beam polarization at $\sqrt{s}=3 \mathrm{TeV}$ and an integrated luminosity of $L_{\text {int }}=2000 \mathrm{fb}{ }^{-1}$ are $[-4.05 ; 3.67] \times 10^{-12} \mathrm{GeV}^{-4},[-3.08 ; 2.12] \times 10^{-12} \mathrm{GeV}^{-4}$, and $[-1.98 ; 0.64] \times 10^{-13} \mathrm{GeV}^{-4}$, which show improvement over the current bounds.
\end{abstract}

\section{Introduction}

The Standard Model (SM) has been proven to be highly successful through many significant experimental tests, in particular the discovery of a new particle consistent with the SM Higgs boson with a mass between $125-126 \mathrm{GeV}$ detected by the ATLAS and the CMS experiments at the LHC $[1$, 2]. On the other hand, since many important questions such as the origin of mass, the large hierarchy between electroweak and the Planck scale, the strong CP problem, and the matter/antimatter asymmetry remain unanswered in the SM, we need to study physics beyond the SM. One of the ways of probing new physics beyond the SM is to investigate the anomalous gauge boson interactions. Gauge boson self-interactions in the SM are exactly described by the $S U_{L}(2) \times U_{Y}(1)$ gauge symmetry. The precision measurements of gauge boson self-interactions can further verify the SM. Furthermore, the existence of anomalous gauge boson couplings may be a sign of new physics beyond the SM. The effective Lagrangian approach is one of the common ways for searching new physics beyond the SM in a model independent way. In particular, the anomalous quartic gauge boson couplings can be examined with the aid of the effective Lagrangian approach. Such an approach is parameterized by high-dimensional operators which induce anomalous quartic gauge couplings that modify the interactions between the electroweak gauge bosons.

The LHC is expected to reply some of the fundamental open questions in particle physics. Nevertheless, the analysis of the LHC data is quite difficult due to remnants of the usual proton-proton deep inelastic processes, whereas collisions between electrons and positrons are much simpler to investigate than proton-proton collisions. A linear electron-positron collider with high luminosity and energy is the best option to complement and to expand the LHC physics program. The CLIC is one of the most popular linear colliders, purposed to follow out electron-positron collisions at energies from $0.35 \mathrm{TeV}$ to $3 \mathrm{TeV}$ [3]. To have its high luminosity and energy is quite important with regard to new physics research beyond the SM. Since the anomalous quartic couplings defined through effective Lagrangians have dimension-8, they have very strong energy dependence. Therefore, the anomalous cross section including these vertices has higher energy dependence than the SM cross section. Hence, CLIC will have 
a great potential to examine the anomalous quartic gauge boson couplings.

High-dimensional effective operators describing the anomalous quartic gauge boson couplings are expressed by either linear or nonlinear effective Lagrangians. Nonlinear effective Lagrangians are considered if there is no Higgs boson in the low energy spectrum. However, linear effective Lagrangians are obtained by using a linear representation of gauge symmetry that is broken by the conventional SM Higgs mechanism. It becomes important to study the anomalous quartic gauge boson couplings based on linear effective Lagrangians due to the discovery of a Higgs boson in the LHC. For these reasons, we only deal with dimension-8 operators in our work.

In this paper, we will analyze the anomalous quartic couplings via $e^{+} e^{-} \rightarrow \nu_{e} W^{-} W^{+} \bar{\nu}_{e}$ process with semileptonic decay including polarized electron (positron) beam effects at the CLIC for the center-of-mass energies of 1.4 and $3 \mathrm{TeV}$.

\section{Dimension-Eight Operators for Quartic Gauge Couplings}

There are three classes of operators that describe the anomalous quartic couplings. The first class of operators can be parameterized in terms of only the covariant derivative of the field $D_{\mu} \Phi$. This class includes two independent operators [4]:

$$
\begin{aligned}
& L_{S 0}=\frac{f_{S 0}}{\Lambda^{4}}\left[\left(D_{\mu} \Phi\right)^{\dagger} D_{\nu} \Phi\right]\left[\left(D^{\mu} \Phi\right)^{\dagger} D^{\nu} \Phi\right], \\
& L_{S 1}=\frac{f_{S 1}}{\Lambda^{4}}\left[\left(D_{\mu} \Phi\right)^{\dagger} D_{\mu} \Phi\right]\left[\left(D^{\nu} \Phi\right)^{\dagger} D^{\nu} \Phi\right] .
\end{aligned}
$$

The second class of operators are related to $D_{\mu} \Phi$ and the field strength. These seven operators are given as follows [4]:

$$
\begin{aligned}
& L_{M 0}=\frac{f_{M 0}}{\Lambda^{4}} \operatorname{tr}\left[W_{\mu \nu} W^{\mu \nu}\right]\left[\left(D_{\beta} \Phi\right)^{\dagger} D^{\beta} \Phi\right] \\
& L_{M 1}=\frac{f_{M 1}}{\Lambda^{4}} \operatorname{tr}\left[W_{\mu \nu} W^{\nu \beta}\right]\left[\left(D_{\beta} \Phi\right)^{\dagger} D^{\mu} \Phi\right] \\
& L_{M 2}=\frac{f_{M 2}}{\Lambda^{4}}\left[B_{\mu \nu} B^{\mu \nu}\right]\left[\left(D_{\beta} \Phi\right)^{\dagger} D^{\beta} \Phi\right], \\
& L_{M 3}=\frac{f_{M 3}}{\Lambda^{4}}\left[B_{\mu \nu} B^{\nu \beta}\right]\left[\left(D_{\beta} \Phi\right)^{\dagger} D^{\mu} \Phi\right] \\
& L_{M 4}=\frac{f_{M 4}}{\Lambda^{4}}\left[\left(D_{\mu} \Phi\right)^{\dagger} W_{\beta \nu} D^{\mu} \Phi\right] B^{\beta \nu}, \\
& L_{M 5}=\frac{f_{M 5}}{\Lambda^{4}}\left[\left(D_{\mu} \Phi\right)^{\dagger} W_{\beta \nu} D^{\nu} \Phi\right] B^{\beta \mu}, \\
& L_{M 6}=\frac{f_{M 6}}{\Lambda^{4}}\left[\left(D_{\mu} \Phi\right)^{\dagger} W_{\beta \nu} W^{\beta \nu} D^{\mu} \Phi\right], \\
& L_{M 7}=\frac{f_{M 7}}{\Lambda^{4}}\left[\left(D_{\mu} \Phi\right)^{\dagger} W_{\beta \nu} W^{\beta \mu} D^{\nu} \Phi\right]
\end{aligned}
$$

The remaining operators contain, solely, the field strength tensors. These operators can be expressed as [4]

$$
L_{T 0}=\frac{f_{T 0}}{\Lambda^{4}} \operatorname{tr}\left[W_{\mu \nu} W^{\mu \nu}\right] \operatorname{tr}\left[W_{\alpha \beta} W^{\alpha \beta}\right],
$$

$$
\begin{aligned}
& L_{T 1}=\frac{f_{T 1}}{\Lambda^{4}} \operatorname{tr}\left[W_{\alpha \nu} W^{\mu \beta}\right] \operatorname{tr}\left[W_{\mu \beta} W^{\alpha \nu}\right] \\
& L_{T 2}=\frac{f_{T 2}}{\Lambda^{4}} \operatorname{tr}\left[W_{\alpha \mu} W^{\mu \beta}\right] \operatorname{tr}\left[W_{\beta \nu} W^{\nu \alpha}\right] \\
& L_{T 5}=\frac{f_{T 5}}{\Lambda^{4}} \operatorname{tr}\left[W_{\mu \nu} W^{\mu \nu}\right] B_{\alpha \beta} B^{\alpha \beta}, \\
& L_{T 6}=\frac{f_{T 6}}{\Lambda^{4}} \operatorname{tr}\left[W_{\alpha \nu} W^{\mu \beta}\right] B_{\mu \beta} B^{\alpha \nu}, \\
& L_{T 7}=\frac{f_{T 7}}{\Lambda^{4}} \operatorname{tr}\left[W_{\alpha \mu} W^{\mu \beta}\right]\left[B_{\beta \nu} B^{\nu \alpha}\right] \\
& L_{T 8}=\frac{f_{T 8}}{\Lambda^{4}} B_{\mu \nu} B^{\mu \nu} B_{\alpha \beta} B^{\alpha \beta} \\
& L_{T 9}=\frac{f_{T 9}}{\Lambda^{4}} B_{\alpha \mu} B^{\mu \beta} B_{\beta \nu} B^{\nu \alpha} .
\end{aligned}
$$

The complete list of quartic vertices modified by these operators is given in Table 1.

There are total 59 Feynman diagrams for process $e^{+} e^{-} \rightarrow$ $\nu_{e} W^{+} W^{-} \bar{\nu}_{e}$ including anomalous quartic $W W W W, W W Z \gamma$, and $W W Z Z$ couplings. The three diagrams in presence of anomalous quartic $W W W W, W W Z \gamma$, and $W W Z Z$ couplings are shown in Figure 1. We can see from Table 1 that while $L_{S 0}$ and $L_{S 1}$ operators modify the anomalous $W W W W$ and $W W Z Z$ couplings, dimension- 8 effective $L_{T 0}$ operator causes the anomalous $W W W W, W W Z \gamma$, and $W W Z Z$ vertices.

There have been many studies for the anomalous gauge self-interactions at linear and hadron colliders. On the other hand, the anomalous quartic couplings arising from dimension-8 operators at the LHC and the future hadron colliders have been investigated in [5-39]. In our paper, we choose $L_{S 0}, L_{S 1}$, and $L_{T 0}$ operators to investigate anomalous quartic couplings. In the literature, these couplings have been examined three different $f_{S 0} / \Lambda^{4}, f_{S 1} / \Lambda^{4}$, and $f_{T 0} / \Lambda^{4}$ couplings. Reference [5] has been experimentally obtained the bounds on the $f_{S 0} / \Lambda^{4}$ and $f_{S 1} / \Lambda^{4}$ using proton-proton collision data corresponding to an integrated luminosity of $20.3 \mathrm{fb}^{-1}$ at a center-of-mass energy of $8 \mathrm{TeV}$ collected by the ATLAS detector at the LHC. In [6], the sensitivity bounds are obtained only on $f_{S 0} / \Lambda^{4}$ and $f_{S 1} / \Lambda^{4}$ at $99 \%$ confidence level (CL) for $\sqrt{s}=14 \mathrm{TeV}$ via $p p \rightarrow j j e^{ \pm} \mu^{ \pm} \nu \nu$ process. Similarly the sensitivity bounds on $f_{T 0} / \Lambda^{4}$ with $95 \% \mathrm{CL}$ via triboson production at the proton-proton colliders for $\sqrt{s}=14 \mathrm{TeV}$ are obtained in [8]. In [7], the $f_{S 0} / \Lambda^{4}, f_{S 1} / \Lambda^{4}$, and $f_{T 0} / \Lambda^{4}$ couplings have been studied via $W W W$ final state with full leptonic decay and semileptonic decay for $\sqrt{s}=14 \mathrm{TeV}$ at the LHC and $\sqrt{s}=100 \mathrm{TeV}$ for future hadron collider. The best available constraints on $f_{S 0} / \Lambda^{4}$, $f_{S 1} / \Lambda^{4}$, and $f_{T 0} / \Lambda^{4}$ parameters defining these anomalous quartic couplings obtained from one parameter analysis in [5-8] are summarized in Table 2. In [39] the authors examine the nonlinear parametrization through the processes $p p \rightarrow$ $l^{ \pm} v_{l} l^{ \pm} v_{l} j j$ and $p p \rightarrow l^{ \pm} v_{l} l^{\mp} v_{l} j j$ at the LHC. 


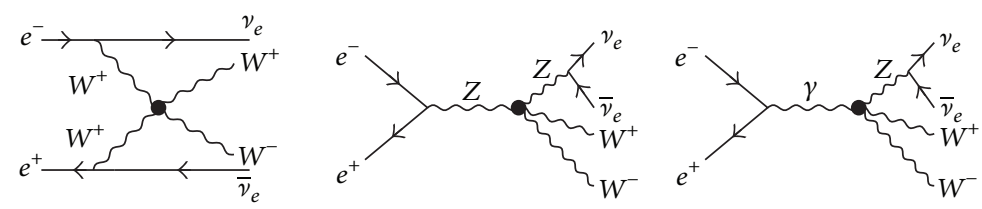

FIGURE 1: The Feynman diagrams for the $e^{+} e^{-} \rightarrow v_{e} W^{-} W^{+} \bar{\nu}_{e}$ process in the presence of anomalous quartic $W W W W, W W Z \gamma$, and $W W Z Z$ couplings.

TABLE 1: Quartic vertices modified by each dimension-8 operator are marked with X [4].

\begin{tabular}{|c|c|c|c|c|c|c|c|c|c|}
\hline & $W W W W$ & $W W Z Z$ & $Z Z Z Z$ & WWAZ & WWAA & $Z Z Z A$ & $Z Z A A$ & $Z A A A$ & $A A A A$ \\
\hline$\overline{L_{S 0}, L_{S 1}}$ & $\mathrm{X}$ & $\mathrm{X}$ & $\mathrm{X}$ & & & & & & \\
\hline$L_{M 0}, L_{M 1}, L_{M 6}, L_{M 7}$ & $\mathrm{X}$ & $\mathrm{X}$ & $\mathrm{X}$ & $\mathrm{X}$ & $\mathrm{X}$ & $\mathrm{X}$ & $\mathrm{X}$ & & \\
\hline$L_{M 2}, L_{M 3}, L_{M 4}, L_{M 5}$ & & $\mathrm{X}$ & $\mathrm{X}$ & $\mathrm{X}$ & $\mathrm{X}$ & $\mathrm{X}$ & $\mathrm{X}$ & & \\
\hline$L_{T 0}, L_{T 1}, L_{T 2}$ & $\mathrm{X}$ & $\mathrm{X}$ & $\mathrm{X}$ & $\mathrm{X}$ & $\mathrm{X}$ & $\mathrm{X}$ & $\mathrm{X}$ & $\mathrm{X}$ & $\mathrm{X}$ \\
\hline$L_{T 5}, L_{T 6}, L_{T 7}$ & & $\mathrm{X}$ & $\mathrm{X}$ & $\mathrm{X}$ & $\mathrm{X}$ & $\mathrm{X}$ & $\mathrm{X}$ & $\mathrm{X}$ & $\mathrm{X}$ \\
\hline$L_{T 8}, L_{T 9}$ & & & $\mathrm{X}$ & & & $\mathrm{X}$ & $\mathrm{X}$ & $\mathrm{X}$ & $\mathrm{X}$ \\
\hline
\end{tabular}

\section{Numerical Analysis}

The important features of a linear collider are its clean experimental environment, high energy, and polarized beams. A polarized electron beam would provide suitable platform searching of the SM and for diagnosing new physics. Observation of even the tiniest signal which conflicts with the SM expectations would be a convincing evidence for physics beyond the SM. Proper selections of the electron and positron beam polarization may therefore be used to enhance the new physics signal and also to considerably suppress backgrounds.

We use MadGraph5 [40] to generate the signal and background events with the effective Lagrangian implemented through FEYNRULES [41]. In order to probe the sensitivity of anomalous quartic couplings $\left(L_{S 0}, L_{S 1}\right.$, and $\left.L_{T 0}\right)$, we analyzed the process $e^{+} e^{-} \rightarrow W^{+}\left(\rightarrow l^{+} \nu\right) W^{-}(\rightarrow$ jets $) v_{e} \bar{\nu}_{e}$. In our analysis the main background processes yield identical final states to the signal process. Here we assume that one of the W's in the final state decays into leptonic channel and the other one decays into hadronic channel. Therefore, the final state signal and background topology of the process consists of an energetic lepton ( $l$ ), neutrinos (missing $E_{T}$ ), and two hadronic jets $(2 j)$. We apply following set of cuts in order to suppress the backgrounds and enhance the signal for the anomalous quartic interactions in $e^{+} e^{-} \rightarrow W^{+} W \nu_{e} \bar{\nu}_{e}$ process:

(1) $P_{T j}>10 \mathrm{GeV},\left|\eta_{j}\right|<5$,

(2) $E_{\text {miss }}>10 \mathrm{GeV}$ (missing $E_{T}$ (sum of neutrino's momenta)),

(3) $\left|M_{j j}-M_{W}\right|<15 \mathrm{GeV}$,

(4) $\Delta R>0.4$,

where $j=u, d, s, c$ and $\Delta R$ is the angular separation between any two objects. $\Delta R=\sqrt{\Delta \varphi^{2}+\Delta \eta^{2}}$ with $\varphi$ representing the azimuthal angle with respect to the beam directions.

If the electroweak nature of the interactions of the processes is taken into account, for a process with electron and positron beam polarization, the cross section can be expressed as [42]

$$
\begin{aligned}
\sigma= & \frac{1}{4}\left(1-P_{e^{+}}\right)\left(1+P_{e^{-}}\right) \sigma_{-1+1} \\
& +\frac{1}{4}\left(1+P_{e^{+}}\right)\left(1-P_{e^{-}}\right) \sigma_{+1-1} .
\end{aligned}
$$

Here $\sigma_{a b}$ represents the calculated cross section with fixed helicities $a$ for positron and $b$ for the electron. $P_{e^{-}}$is the electron beam and $P_{e^{+}}$is the positron relative polarization. It may be noted that the considered process includes only a weak interaction. Therefore, only left-handed electrons (righthanded positrons) should be taken into account because of the structure of the $W e^{-} v_{e}\left(W e^{+} \bar{v}_{e}\right)$ vertex. Hence, the leftpolarized electron (right-polarized positron) beam would enhance the cross section. This effect can be seen in Figures 2-7 for various polarization schemes. The total cross section of the $e^{+} e^{-} \rightarrow v_{e} W^{-} W^{+} \bar{\nu}_{e}$ process as a function of $f_{S 0} / \Lambda^{4}$ coupling for 1.4 and $3 \mathrm{TeV}$ center-of-mass energies is shown in Figures 2 and 3, respectively. Figures 4 and 5 depict the total cross section depending on the anomalous $f_{S 1} / \Lambda^{4}$ coupling at $\sqrt{s}=1.4 \mathrm{TeV}$ and $\sqrt{s}=3 \mathrm{TeV}$ energies, respectively. The total cross sections are plotted in Figures 6 and 7 with respect to $f_{T 0} / \Lambda^{4}$ coupling for the center-of-mass energies of $1.4 \mathrm{TeV}$ and $3 \mathrm{TeV}$, respectively. As can be seen from Figures 2 and 7 , the polarization $\left(P_{e^{-}}=-80 \% ; P_{e^{+}}=+60 \%\right)$ enhances the cross sections the most compared to the other considered to polarization schemes. The lowest points of the curves in Figures 2 and 7 correspond the value of the SM cross section. As seen from the figures, the increase of the center-of-mass energy leads to remarkable enhancement of the deviations from the SM. For instance, in Figure 2 cross section increases nearly by a factor of 10 from the SM for $f_{S 0} / \Lambda^{4}=1 \times$ $10^{-9} \mathrm{GeV}^{-4}$ at $1.4 \mathrm{TeV}$. However, cross section increases nearly by a factor of 100 at $3 \mathrm{TeV}$ for the same value for $f_{S 0} / \Lambda^{4}$. On the other hand, the obtained cross sections are very sensitive to the anomalous parameters. For instance, in Figure 3 cross section increases two orders of magnitude as $f_{S 1} / \Lambda^{4}$ increases 
TABLE 2: Current sensitivity bounds on anomalous parameters for $95 \% \mathrm{CL}$ with $20.3 \mathrm{fb}^{-1}$ for $\sqrt{s}=8 \mathrm{TeV}$ [5], 99\% CL with $100 \mathrm{fb}^{-1}$ for $\sqrt{s}=14 \mathrm{TeV}$ [6], 95\% CL with $100 \mathrm{fb}^{-1}$ for $\sqrt{s}=14 \mathrm{TeV}$ [7], $5 \sigma$ with $300 \mathrm{fb}^{-1}$ for $\sqrt{s}=14 \mathrm{TeV}$ [8], and 95\% CL with $3000 \mathrm{fb}^{-1}$ for $\sqrt{s}=100 \mathrm{TeV}$ [7].

\begin{tabular}{lcccc}
\hline$L\left(\mathrm{fb}^{-1}\right)$ & $\sqrt{s}(\mathrm{TeV})$ & $f_{S 0} / \Lambda^{4}\left(\mathrm{GeV}^{-4}\right)$ & $f_{S 1} / \Lambda^{4}\left(\mathrm{GeV}^{-4}\right)$ & $f_{T 0} / \Lambda^{4}\left(\mathrm{GeV}^{-4}\right)$ \\
\hline $20.3[5]$ & 8 & {$[-0.13 ; 0.18] \times 10^{-8}$} & {$[-0.25 ; 0.31] \times 10^{-8}$} & - \\
$100[6]$ & 14 & {$[-2.2 ; 2.4] \times 10^{-11}$} & {$[-2.5 ; 2.5] \times 10^{-11}$} & - \\
$100[7]$ & 14 & {$[-1.8 ; 1.8] \times 10^{-10}$} & {$[-2.7 ; 2.8] \times 10^{-10}$} & {$[-5.8 ; 5.9] \times 10^{-13}$} \\
$300[8]$ & 14 & - & - & {$[-1.2 ; 1.2] \times 10^{-12}$} \\
$3000[7]$ & 100 & {$[-2.9 ; 3.0] \times 10^{-12}$} & {$[-1.3 ; 1.1] \times 10^{-12}$} & {$[-3.7 ; 3.0] \times 10^{-15}$} \\
\hline
\end{tabular}

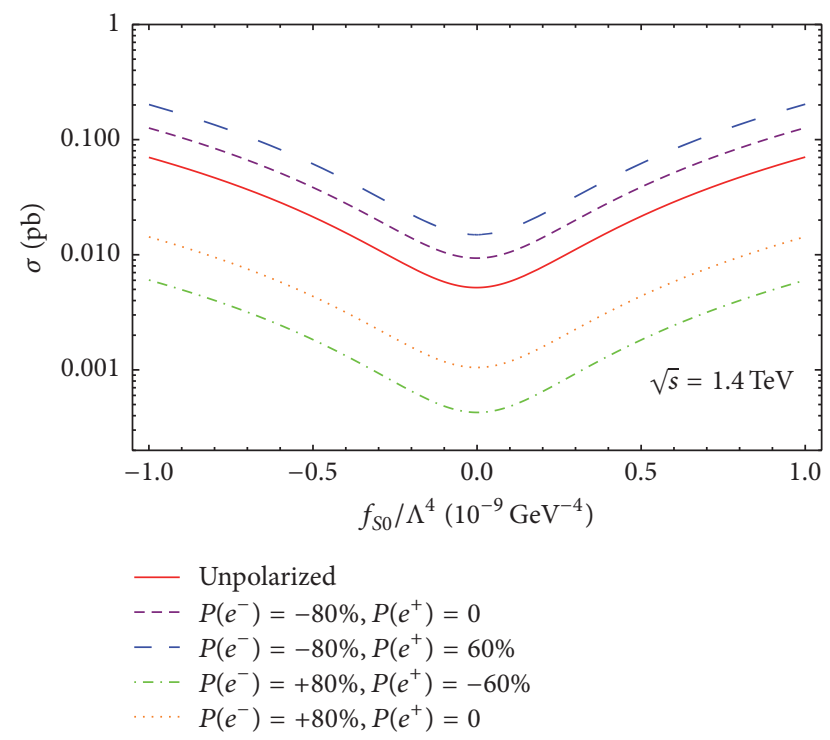

Figure 2: The total cross section for $e^{+} e^{-} \rightarrow \nu_{e} W^{-} W^{+} \bar{\nu}_{e}$ processes as a function of $f_{S 0} / \Lambda^{4}$ at the $\sqrt{s}=1.4 \mathrm{TeV}$ for different polarization of the positron and electron beams.

from 0 to $1 \times 10^{-9} \mathrm{GeV}^{-4}$. As seen from these figures, the total cross sections depend on the center-of-mass energy. When the center-of-mass energies are changed from $1.4 \mathrm{TeV}$ to $3 \mathrm{TeV}$, the cross sections nearly increase by a factor of 100 . The cross sections are almost symmetric with respect to change in the sign of anomalous couplings. Therefore, main contribution comes from the quadratic anomalous couplings terms.

In order to examine the sensitivity to the anomalous couplings, we use one parameter $\chi^{2}$ criterion without systematic error. The $\chi^{2}$ function is defined as follows:

$$
\chi^{2}=\left(\frac{\sigma_{\mathrm{SM}}-\sigma_{N P}}{\sigma_{\mathrm{SM}} \delta_{\text {stat }}}\right)^{2},
$$

where $\sigma_{N P}$ is the total cross section including SM and new physics, $\delta_{\text {stat }}=1 / \sqrt{N}$ is the statistical error, and $N$ is the number of background events $N=L_{\text {int }} \sigma_{\mathrm{SM}}$, where $L_{\text {int }}$ is the integrated CLIC luminosity. We have obtained 95\% CL limits on the anomalous coupling parameters using this analyze method at $\sqrt{s}=1.4 \mathrm{TeV}$ and $3 \mathrm{TeV}$ for different integrated luminosity values and final polarization configurations. Polarization improves the sensitivity bound of anomalous parameters as seen from Tables 3-8. As we expected,



Figure 3: The total cross section for $e^{+} e^{-} \rightarrow \nu_{e} W^{-} W^{+} \bar{\nu}_{e}$ processes as a function of $f_{S 0} / \Lambda^{4}$ at the $\sqrt{s}=3 \mathrm{TeV}$ for different polarization of the positron and electron beams.

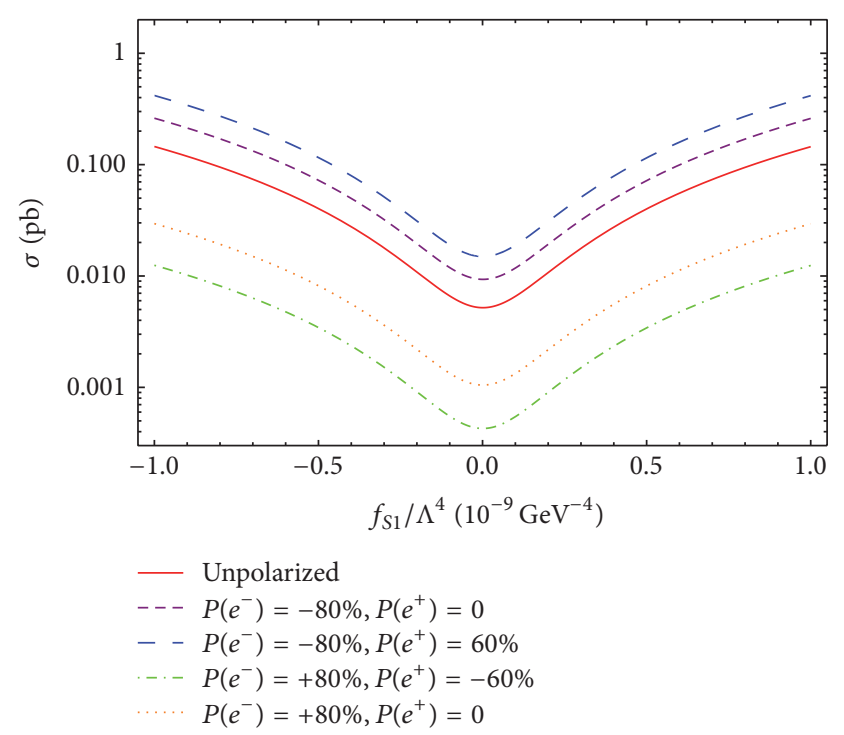

Figure 4: The total cross section for $e^{+} e^{-} \rightarrow \nu_{e} W^{-} W^{+} \bar{\nu}_{e}$ processes as a function of $f_{S 1} / \Lambda^{4}$ at the $\sqrt{s}=1.4 \mathrm{TeV}$ for different polarization of the positron and electron beams. 




Figure 5: The total cross section for $e^{+} e^{-} \rightarrow \nu_{e} W^{-} W^{+} \bar{\nu}_{e}$ processes as a function of $f_{S 1} / \Lambda^{4}$ at the $\sqrt{s}=3 \mathrm{TeV}$ for different polarization of the positron and electron beams.

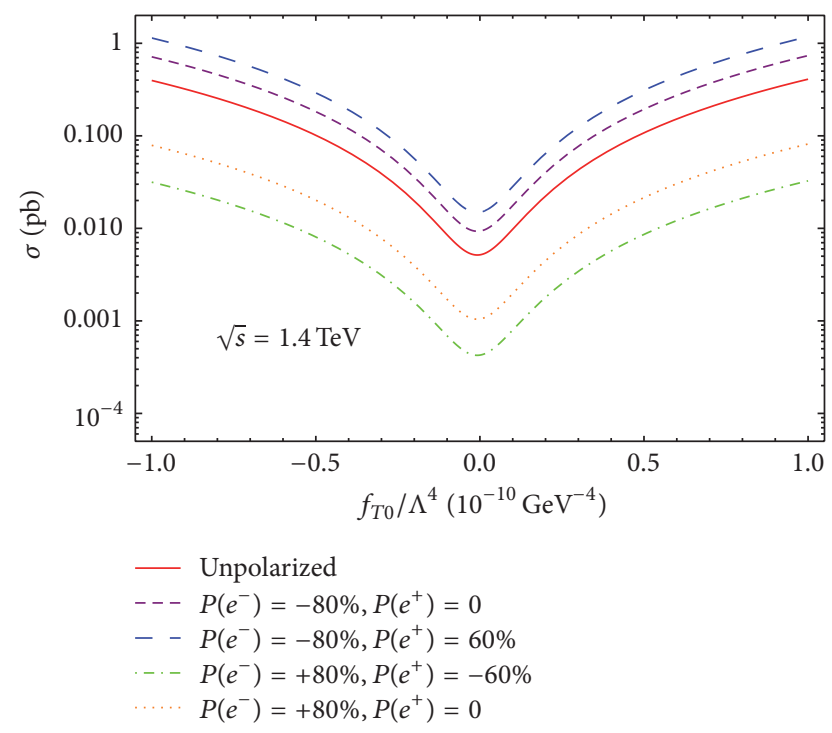

Figure 6: The total cross section for $e^{+} e^{-} \rightarrow \nu_{e} W^{-} W^{+} \bar{\nu}_{e}$ processes as a function of $f_{T 0} / \Lambda^{4}$ at the $\sqrt{s}=1.4 \mathrm{TeV}$ for different polarization of the positron and electron beams.

the best limits are obtained for the $P_{e^{-}}=-80 \% ; P_{e^{+}}=60 \%$ polarization state.

The expected best sensitivities on $f_{S 0} / \Lambda^{4}$ and $f_{S 1} / \Lambda^{4}$ couplings in Tables 3-6 are far beyond the sensitivities of the LHC. Also, we observe that our limits for $f_{S 0} / \Lambda^{4}$ in Table 3 at $\sqrt{s}=1.4 \mathrm{TeV}$ are competitive with the results in [6] and one order of magnitude better than the ones reported for the LHC with $L=100 \mathrm{fb}^{-1}$ by [7]. Additionally, our limits for $\sqrt{s}=$ $3 \mathrm{TeV}$ are at the same order of magnitude with the LHC results for the $L=3000 \mathrm{fb}^{-1}$ and $\sqrt{s}=100 \mathrm{TeV}$. These results can be seen if Table 2 is compared to Table 4. Similar interpretations can be made for our bounds on $f_{S 1} / \Lambda^{4}$ from Tables 5 and 6 .

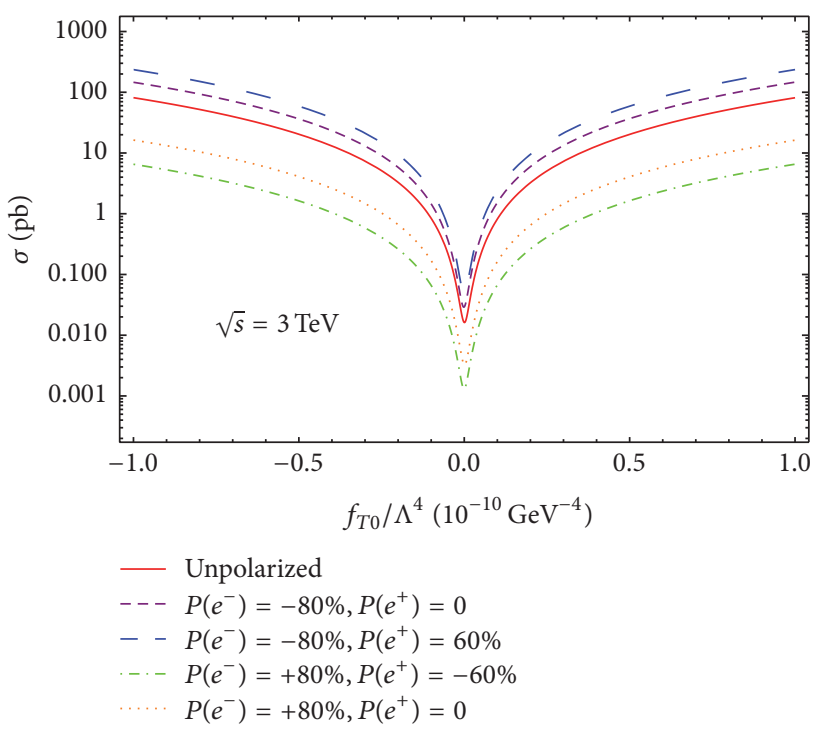

Figure 7: The total cross section for $e^{+} e^{-} \rightarrow \nu_{e} W^{-} W^{+} \bar{\nu}_{e}$ processes as a function of $f_{T 0} / \Lambda^{4}$ at the $\sqrt{s}=3 \mathrm{TeV}$ for different polarization of the positron and electron beams.

In addition, we can see from Table 7 that the limits on $f_{T 0} / \Lambda^{4}$ for $\sqrt{s}=1.4 \mathrm{TeV}$ are very close to the LHC bounds for $5 \sigma$ with $300 \mathrm{fb}^{-1}$ for $\sqrt{s}=14 \mathrm{TeV}$. However, the bounds for $\sqrt{s}=100 \mathrm{TeV}$ future hadron collider with $L=3000 \mathrm{fb}^{-1}$ are better than our limits on $f_{T 0} / \Lambda^{4}$ as stated from Table 8 . In the meantime, the relation between linear and nonlinear parametrization is shown in [4] as the following formations:

$$
\begin{aligned}
\alpha_{4} & =\frac{f_{S 0}}{\Lambda^{4}} \frac{v^{4}}{8}, \\
\alpha_{4}+2 \alpha_{5} & =\frac{f_{S 1}}{\Lambda^{4}} \frac{v^{4}}{8} .
\end{aligned}
$$

In [39] the authors study the nonlinear parametrization through the processes $p p \rightarrow l^{ \pm} v_{l} l^{ \pm} v_{l} j j$ and $p p \rightarrow l^{ \pm} v_{l} l^{\mp} v_{l} j j$ at the LHC as mentioned above. This process can isolate the $W W W W$ vertex. The best 95\% CL bounds they have found are $\alpha_{4}=[-0.0011 ; 0.0016]$ and $\alpha_{5}=[-0.0022 ; 0.0016]$ for the $\sqrt{s}=14 \mathrm{TeV}$ and $L=3 \mathrm{ab}^{-1}$. By using (6), these limits can be converted to linear parametrization. The results obtained in this case are $f_{S 0} / \Lambda^{4}=\left[-2.40 \times 10^{-12} ; 3.50 \times 10^{-12}\right] \mathrm{GeV}^{-4}$ and $f_{S 1} / \Lambda^{4}=\left[-1.20 \times 10^{-11} ; 1.75 \times 10^{-11}\right] \mathrm{GeV}^{-4}$. As seen from the Tables 4 and 6 , these limits are of the same order as our best limits for the $f_{S 0} / \Lambda^{4}$, but the best limits we obtain for $f_{S 1} / \Lambda^{4}$ are one order of magnitude better. Additionally, when the $10 \%$ systematic uncertainty factor is taken into account, the sensitivity of our best obtained bounds decreases by about one order magnitude for $f_{S 0} / \Lambda^{4}$ and $f_{S 1} / \Lambda^{4}$ and 3time decrease for $f_{T 0} / \Lambda^{4}$ coupling.

\section{Conclusions}

The CLIC is a high energy collider which has TeV scale energy and very high luminosity. Particularly, operating with its high energy and luminosity is extremely important in order 
TABLE 3: Sensitivity of $f_{S 0} / \Lambda^{4}$ at $95 \%$ CL for $\sqrt{s}=1.4 \mathrm{TeV}$ in units of $\mathrm{GeV}^{-4}$.

\begin{tabular}{lccr}
\hline$L\left(\mathrm{fb}^{-1}\right)$ & Unpolarized & $P_{e^{-}}=-80 \% ; P_{e^{+}}=0$ & $P_{e^{-}}=-80 \% ; P_{e^{+}}=60$ \\
\hline 50 & {$[-10.02 ; 9.67] \times 10^{-11}$} & {$[-8.63 ; 8.39] \times 10^{-11}$} & {$[-7.72 ; 7.70] \times 10^{-11}$} \\
250 & {$[-6.76 ; 6.41] \times 10^{-11}$} & {$[-5.81 ; 5.57] \times 10^{-11}$} & {$[-5.22 ; 4.90] \times 10^{-11}$} \\
500 & {$[-5.71 ; 5.36] \times 10^{-11}$} & {$[-4.91 ; 4.67] \times 10^{-11}$} & {$[-4.41 ; 4.09] \times 10^{-11}$} \\
750 & {$[-5.18 ; 4.83] \times 10^{-11}$} & {$[-4.44 ; 4.21] \times 10^{-11}$} & {$[-4.00 ; 3.69] \times 10^{-11}$} \\
1000 & {$[-4.83 ; 4.48] \times 10^{-11}$} & {$[-4.14 ; 3.90] \times 10^{-11}$} & {$[-3.74 ; 3.42] \times 10^{-11}$} \\
1250 & {$[-4.58 ; 4.23] \times 10^{-11}$} & {$[-3.93 ; 3.69] \times 10^{-11}$} & {$[-3.54 ; 3.23] \times 10^{-11}$} \\
1500 & {$[-4.38 ; 4.04] \times 10^{-11}$} & {$[-3.76 ; 3.52] \times 10^{-11}$} & {$[-3.39 ; 3.08] \times 10^{-11}$} \\
\hline
\end{tabular}

TABLE 4: Sensitivity of $f_{S 0} / \Lambda^{4}$ at $95 \% \mathrm{CL}$ for $\sqrt{s}=3 \mathrm{TeV}$ in units of $\mathrm{GeV}^{-4}$.

\begin{tabular}{lccc}
\hline$L\left(\mathrm{fb}^{-1}\right)$ & Unpolarized & $P_{e^{-}}=-0.80 \% ; P_{e^{+}}=0$ & $P_{e^{-}}=-0.80 \% ; P_{e^{+}}=0.60$ \\
\hline 50 & {$[-12.99 ; 12.27] \times 10^{-12}$} & {$[-11.26 ; 10.56] \times 10^{-12}$} & {$[-9.89 ; 9.51] \times 10^{-12}$} \\
250 & {$[-8.81 ; 8.09] \times 10^{-12}$} & {$[-7.65 ; 6.95] \times 10^{-12}$} & {$[-6.68 ; 6.29] \times 10^{-12}$} \\
400 & {$[-7.88 ; 7.16] \times 10^{-12}$} & {$[-6.84 ; 6.14] \times 10^{-12}$} & {$[-5.96 ; 5.58] \times 10^{-12}$} \\
800 & {$[-6.69 ; 5.96] \times 10^{-12}$} & {$[-5.81 ; 5.11] \times 10^{-12}$} & {$[-5.04 ; 4.66] \times 10^{-12}$} \\
1200 & {$[-6.08 ; 5.36] \times 10^{-12}$} & {$[-5.29 ; 4.59] \times 10^{-12}$} & {$[-4.56 ; 4.19] \times 10^{-12}$} \\
1600 & {$[-5.69 ; 4.96] \times 10^{-12}$} & {$[-4.95 ; 4.25] \times 10^{-12}$} & {$[-4.27 ; 3.89] \times 10^{-12}$} \\
2000 & {$[-5.40 ; 4.67] \times 10^{-12}$} & {$[-4.70 ; 4.00] \times 10^{-12}$} & {$[-4.05 ; 3.67] \times 10^{-12}$} \\
\hline
\end{tabular}

TABLE 5: Sensitivity of $f_{S 1} / \Lambda^{4}$ at $95 \%$ CL for $\sqrt{s}=1.4 \mathrm{TeV}$ in units of $\mathrm{GeV}^{-4}$.

\begin{tabular}{lccc}
\hline$L\left(\mathrm{fb}^{-1}\right)$ & Unpolarized & $P_{e^{-}}=-0.80 \% ; P_{e^{+}}=0$ & $P_{e^{-}}=-0.80 \% ; P_{e^{+}}=0.60$ \\
\hline 50 & {$[-6.82 ; 6.62] \times 10^{-11}$} & {$[-5.92 ; 5.69] \times 10^{-11}$} & {$[-5.26 ; 5.07] \times 10^{-11}$} \\
250 & {$[-4.60 ; 4.39] \times 10^{-11}$} & {$[-3.99 ; 3.77] \times 10^{-11}$} & {$[-3.55 ; 3.36] \times 10^{-11}$} \\
500 & {$[-3.88 ; 3.68] \times 10^{-11}$} & {$[-3.38 ; 3.15] \times 10^{-11}$} & {$[-2.99 ; 2.81] \times 10^{-11}$} \\
750 & {$[-3.52 ; 3.31] \times 10^{-11}$} & {$[-3.06 ; 2.84] \times 10^{-11}$} & {$[-2.72 ; 2.53] \times 10^{-11}$} \\
1000 & {$[-3.28 ; 3.08] \times 10^{-11}$} & {$[-2.86 ; 2.64] \times 10^{-11}$} & {$[-2.54 ; 2.35] \times 10^{-11}$} \\
1250 & {$[-3.11 ; 2.90] \times 10^{-11}$} & {$[-2.71 ; 2.49] \times 10^{-11}$} & {$[-2.40 ; 2.22] \times 10^{-11}$} \\
1500 & {$[-2.98 ; 2.77] \times 10^{-11}$} & {$[-2.59 ; 2.37] \times 10^{-11}$} & {$[-2.30 ; 2.11] \times 10^{-11}$} \\
\hline
\end{tabular}

TABLE 6: Sensitivity of $f_{S 1} / \Lambda^{4}$ at $95 \%$ CL for $\sqrt{s}=3 \mathrm{TeV}$ in units of $\mathrm{GeV}^{-4}$.

\begin{tabular}{lccc}
\hline$L\left(\mathrm{fb}^{-1}\right)$ & Unpolarized & $P_{e^{-}}=-0.80 \% ; P_{e^{+}}=0$ & $P_{e^{-}}=-0.80 \% ; P_{e^{+}}=0.60$ \\
\hline 50 & {$[-8.85 ; 7.99] \times 10^{-12}$} & {$[-7.62 ; 6.87] \times 10^{-12}$} & {$[-6.92 ; 5.97] \times 10^{-12}$} \\
250 & {$[-5.72 ; 5.16] \times 10^{-12}$} & {$[-5.23 ; 4.47] \times 10^{-12}$} & {$[-4.80 ; 3.85] \times 10^{-12}$} \\
400 & {$[-5.08 ; 4.52] \times 10^{-12}$} & {$[-4.70 ; 3.94] \times 10^{-12}$} & {$[-4.33 ; 3.38] \times 10^{-12}$} \\
800 & {$[-4.25 ; 3.69] \times 10^{-12}$} & {$[-4.02 ; 3.26] \times 10^{-12}$} & {$[-3.73 ; 2.77] \times 10^{-12}$} \\
1200 & {$[-3.82 ; 3.26] \times 10^{-12}$} & {$[-3.67 ; 2.91] \times 10^{-12}$} & {$[-3.42 ; 2.47] \times 10^{-12}$} \\
1600 & {$[-3.54 ; 2.98] \times 10^{-12}$} & {$[-3.44 ; 2.69] \times 10^{-12}$} & {$[-3.22 ; 2.27] \times 10^{-12}$} \\
2000 & {$[-3.33 ; 2.77] \times 10^{-12}$} & {$[-3.28 ; 2.52] \times 10^{-12}$} & {$[-3.08 ; 2.12] \times 10^{-12}$} \\
\hline
\end{tabular}

TABLe 7: Sensitivity of $f_{T 0} / \Lambda^{4}$ at 95\% CL for $\sqrt{s}=1.4 \mathrm{TeV}$ in units of $\mathrm{GeV}^{-4}$.

\begin{tabular}{lccc}
\hline$L\left(\mathrm{fb}^{-1}\right)$ & Unpolarized & $P_{e^{-}}=-0.80 \% ; P_{e}^{+}=0$ & $P_{e^{-}}=-0.80 \% ; P_{e^{+}}=0.60$ \\
\hline 50 & {$[-4.86 ; 3.25] \times 10^{-12}$} & {$[-4.30 ; 2.75] \times 10^{-12}$} & {$[-4.04 ; 2.38] \times 10^{-12}$} \\
250 & {$[-3.58 ; 1.97] \times 10^{-12}$} & {$[-3.20 ; 1.65] \times 10^{-12}$} & {$[-3.07 ; 1.40] \times 10^{-12}$} \\
500 & {$[-3.18 ; 1.57] \times 10^{-12}$} & {$[-2.86 ; 1.31] \times 10^{-12}$} & {$[-2.77 ; 1.10] \times 10^{-12}$} \\
750 & {$[-2.98 ; 1.37] \times 10^{-12}$} & {$[-2.69 ; 1.14] \times 10^{-12}$} & {$[-2.61 ; 0.95] \times 10^{-12}$} \\
1000 & {$[-2.85 ; 1.24] \times 10^{-12}$} & {$[-2.58 ; 1.03] \times 10^{-12}$} & {$[-2.52 ; 0.85] \times 10^{-12}$} \\
1250 & {$[-2.76 ; 1.15] \times 10^{-12}$} & {$[-2.50 ; 0.95] \times 10^{-12}$} & {$[-2.45 ; 0.78] \times 10^{-12}$} \\
1500 & {$[-2.68 ; 1.08] \times 10^{-12}$} & {$[-2.44 ; 0.89] \times 10^{-12}$} & {$[-2.40 ; 0.73] \times 10^{-12}$} \\
\hline
\end{tabular}


TABLE 8: Sensitivity of $f_{T 0} / \Lambda^{4}$ at $95 \% \mathrm{CL}$ for $\sqrt{s}=3 \mathrm{TeV}$ in units of $\mathrm{GeV}^{-4}$.

\begin{tabular}{lccc}
\hline$L\left(\mathrm{fb}^{-1}\right)$ & Unpolarized & $P_{e^{-}}=-0.80 \% ; P_{e}^{+}=0$ & $P_{e^{-}}=-0.80 \% ; P_{e^{+}}=0.60$ \\
\hline 50 & {$[-4.44 ; 3.09] \times 10^{-13}$} & {$[-3.91 ; 2.59] \times 10^{-13}$} & {$[-3.58 ; 2.24] \times 10^{-13}$} \\
250 & {$[-3.24 ; 1.90] \times 10^{-13}$} & {$[-2.89 ; 1.57] \times 10^{-13}$} & {$[-2.68 ; 1.34] \times 10^{-13}$} \\
400 & {$[-2.97 ; 1.63] \times 10^{-13}$} & {$[-6.84 ; 6.14] \times 10^{-13}$} & {$[-2.48 ; 1.14] \times 10^{-13}$} \\
800 & {$[-2.64 ; 1.30] \times 10^{-13}$} & {$[-2.38 ; 1.07] \times 10^{-13}$} & {$[-2.24 ; 0.90] \times 10^{-13}$} \\
1200 & {$[-2.47 ; 1.13] \times 10^{-13}$} & {$[-2.24 ; 0.92] \times 10^{-13}$} & {$[-2.11 ; 0.78] \times 10^{-13}$} \\
1600 & {$[-2.37 ; 1.03] \times 10^{-13}$} & {$[-2.15 ; 0.83] \times 10^{-13}$} & {$[-2.04 ; 0.70] \times 10^{-13}$} \\
2000 & {$[-2.29 ; 0.95] \times 10^{-13}$} & {$[-2.09 ; 0.77] \times 10^{-13}$} & {$[-1.98 ; 0.64] \times 10^{-13}$} \\
\hline
\end{tabular}

to investigate the anomalous $f_{S 0} / \Lambda^{4}, f_{S 1} / \Lambda^{4}$, and $f_{T 0} / \Lambda^{4}$ couplings through the process $e^{+} e^{-} \rightarrow \nu_{e} W^{-} W^{+} \bar{\nu}_{e}$. Since the anomalous couplings depend on energy strongly, the cross sections that contain these couplings would have momentum dependence compared to those of the SM. We have found that the contribution of the anomalous quartic couplings to the total cross section increases with increasing center-of-mass energy. Because of the structure of $W e^{-} \nu_{e}\left(W e^{+} \bar{v}_{e}\right)$ vertex, it is found that certain polarization of the beam increases the cross sections. In this respect, we find the better sensitivity for the $P_{e^{-}}=-80 \% ; P_{e^{+}}=60 \%$ polarization state.

As a result, the CLIC with very clean experimental conditions and being free from strong interactions with respect to LHC, high colliding energy, and very high luminosity has a potential advantage over the LHC in studying the anomalous $f_{S 0} / \Lambda^{4}, f_{S 1} / \Lambda^{4}$, and $f_{T 0} / \Lambda^{4}$ couplings.

\section{Competing Interests}

The authors declare that there is no conflict of interests regarding the publication of this paper.

\section{References}

[1] S. Chatrchyan, V. Khachatryan, A. M. Sirunyan et al., "Observation of a new boson at a mass of $125 \mathrm{GeV}$ with the CMS experiment at the LHC," Physics Letters B, vol. 716, no. 1, pp. 30-61, 2012.

[2] G. Aad, T. Abajyan, B. Abbott et al., "Observation of a new particle in the search for the Standard Model Higgs boson with the ATLAS detector at the LHC," Physics Letters B, vol. 716, no. 1, pp. 1-29, 2012.

[3] H. Abramowicz, A. Abusleme, K. Afanaciev et al., "Physics at the CLIC e+e- Linear Collider-input to the snowmass process 2013," https://arxiv.org/abs/1307.5288.

[4] M. Baak, A. Blondel, A. Bodek et al., "Study of electroweak interactions at the energy frontier," https://arxiv.org/abs/1310.6708.

[5] ATLAS Collaboration, "Search for triboson $W^{ \pm} W^{ \pm} W^{\mp}$ production in $p p$ collisions at $\sqrt{s}=8 \mathrm{TeV}$ with the ATLAS detector," https://arxiv.org/abs/1610.05088.

[6] O. J. P. Eboli, M. C. Gonzalez-Garcia, and J. K. Mizukoshi, " $p p \rightarrow j j e^{ \pm} \mu^{ \pm} v v$ and $j j e^{ \pm} \mu^{\mp} v v$ at $\mathcal{O}\left(\alpha_{\mathrm{em}}^{6}\right)$ and $\mathcal{O}\left(\alpha_{\mathrm{em}}^{4} \alpha_{s}^{2}\right)$ for the study of the quartic electroweak gauge boson vertex at CERN LHC," Physical Review D, vol. 74, no. 7, Article ID 073005, 2006.

[7] Y. Wen, H. Qu, D. Yang, Q. S. Yan, Q. Li, and Y. Mao, "Probing triple-W production and anomalous WWWW coupling at the CERN LHC and future $100 \mathrm{TeV}$ proton-proton collider," Journal of High Energy Physics, vol. 25, article 1503, 2015.
[8] C. Degrande, J. L. Holzbauer, S.-C. Hsu et al., "Studies of vector Boson scattering and triboson production with DELPHES parametrized fast simulation for snowmass 2013," https://arxiv .org/abs/1309.7452.

[9] I. Şahin, "WZ production at $e \gamma$ colliders and anomalous quartic WWZ $\gamma$ coupling," Journal of Physics G: Nuclear and Particle Physics, vol. 35, no. 3, Article ID 035006, 2008.

[10] İ. Şahin, "Anomalous quartic WW $\gamma \gamma$ and WWZ $\gamma$ couplings through $\mathrm{W}+\mathrm{W}-\mathrm{Z}$ production in $\gamma \gamma$ colliders," Journal of Physics G: Nuclear and Particle Physics, vol. 36, no. 7, Article ID 075007, 2009.

[11] S. Atağ and İ. Şahin, "Anomalous quartic $W W_{\gamma \gamma}$ and $Z Z_{\gamma \gamma}$ couplings in $e_{\gamma}$ collision with initial beam and final state polarizations," Physical Review D, vol. 75, no. 7, Article ID 073003, 2007.

[12] A. Senol, "Anomalous quartic WW $\gamma \gamma$ and ZZ $\gamma \gamma$ couplings in $\gamma \mathrm{p}$ collision at the LHC," International Journal of Modern Physics A, vol. 29, no. 26, Article ID 1450148, 2014.

[13] M. Köksal and A. Senol, "Search for anomalous quartic WWZ $\gamma$ couplings at the future linear $\mathrm{e}^{+} \mathrm{e}^{-}$collider," International Journal of Modern Physics A, vol. 30, no. 20, Article ID 1550107, 2015.

[14] A. Senol and M. Köksal, "Probe of anomalous quartic WWZ $\gamma$ couplings in the photon-photon collisions," Journal of High Energy Physics, vol. 2015, no. 3, article 139, 2015.

[15] A. Senol and M. Köksal, "Analysis of anomalous quartic $W W Z \gamma$ couplings in $\gamma p$ collision at the LHC," Physics Letters B, vol. 742, pp. 143-148, 2015.

[16] M. Köksal, "Anomalous quartic ZZ $\gamma \gamma$ couplings at the CLIC", European Physical Journal Plus, vol. 130, article 75, 2015.

[17] M. Köksal, "Study of anomalous WW $\gamma \gamma$ coupling sensitivity at the compact linear collider," Modern Physics Letters A, vol. 29, no. 34, Article ID 1450184, 2014.

[18] İ. Şahin and A. A. Billur, "Anomalous," Physical Review D, vol. 83, no. 3, 2011.

[19] I. Sahin and B. Sahin, "Anomalous quartic $Z Z_{\gamma \gamma}$ couplings in $\gamma p$," Physical Review D, vol. 86, no. 11, Article ID 115001, 2012.

[20] V. Arı, A. A. Billur, S. İnan, and M. Köksal, "Anomalous $W W \gamma$ couplings with beam polarization at the Compact Linear Collider," Nuclear Physics B, vol. 906, pp. 211-230, 2016.

[21] A. Gutiérrez-Rodríguez, C. Honorato, J. Montaño, and M. Pérez, "Limits on the quartic couplings $Z_{\gamma \gamma \gamma}$ and $Z Z_{\gamma \gamma}$ from $\mathrm{e}^{+}$ $\mathrm{e}^{-}$colliders," Physical Review D, vol. 89, no. 3, Article ID 034003, 2014.

[22] K. Ye, D. Yang, and Q. Li, "CERN LHC sensitivity on measuring $W^{ \pm} Z_{\gamma}$ production and anomalous $W W Z_{\gamma}$ " Physical Review D, vol. 88, no. 1, Article ID 015023, 2013.

[23] O. J. P. Éboli, M. C. Gonzaléz-García, and S. F. Novaes, "Quartic anomalous couplings in e $\gamma$ colliders," Nuclear Physics B, vol. 411, no. 2-3, pp. 381-396, 1994. 
[24] O. J. Éboli, M. B. Magro, P. G. Mercadante, and S. F. Novaes, "Quartic anomalous couplings in $\gamma \gamma$ colliders," Physical Review $D$, vol. 52, no. 1, pp. 15-21, 1995.

[25] G. Bélanger, F. Boudjema, Y. Kurihara, D. Perret-Gallix, and A. Semenov, "Bosonic quartic couplings at LEP2," The European Physical Journal C, vol. 13, no. 3, pp. 283-293, 2000.

[26] W. J. Stirling and A. Werthenbach, "Anomalous quartic couplings in $W^{+} W^{-} \gamma, Z^{0} Z^{0} \gamma$ and $Z^{0} \gamma \gamma$ production at present and future $e^{+} e^{-}$," The European Physical Journal C, vol. 14, p. 103, 2000.

[27] G. A. Leil and W. J. Stirling, "Anomalous quartic couplings in $\mathrm{W}^{+} \mathrm{W}^{-}$gamma production at $\mathrm{e}^{+} \mathrm{e}^{-}$colliders," Journal of Physics G: Nuclear and Particle Physics, vol. 21, no. 4, article 003, pp. 517-524, 1995.

[28] P. J. Dervan, A. Signer, W. J. Stirling, and A. Werthenbach, "Anomalous triple and quartic gauge boson couplings," Journal of Physics G: Nuclear and Particle Physics, vol. 26, no. 5, pp. 607$615,2000$.

[29] W. J. Stirling and A. Werthenbach, "Anomalous quartic couplings in $\nu \bar{\gamma} \gamma \gamma$ production via $W W$-fusion at LEP2," Physics Letters B, vol. 466, no. 2-4, pp. 369-374, 1999.

[30] M. Beyer, W. Kilian, P. Krstonošić et al., "Determination of new electroweak parameters at the ILC—sensitivity to new physics," The European Physical Journal C, vol. 48, no. 2, pp. 353-388, 2006.

[31] T. Pierzchała and K. Piotrzkowski, "Sensitivity to anomalous quartic gauge couplings in photon-photon interactions at the LHC," Nuclear Physics B - Proceedings Supplements, vol. 179-180, pp. 257-264, 2008.

[32] E. Chapon, C. Royon, and O. Kepka, "Anomalous quartic $\mathrm{WW} \gamma \gamma, \mathrm{ZZ} \gamma \gamma$, and trilinear WW $\gamma$ couplings in two-photon processes at high luminosity at the LHC," Physical Review D, vol. 81, no. 7, Article ID 074003, 2010.

[33] J. Shu and J. Yepes, "Left-right non-linear dynamical higgs," Communications in Theoretical Physics, vol. 66, no. 6, p. 643, 2016.

[34] D. R. Green, P. Meade, and M. A. Pleier, "Multi-boson interactions at the run 1 LHC," https://arxiv.org/abs/1610.07572.

[35] G. Aad, E. Abat, J. Abdallah et al., "Evidence of $W_{\gamma \gamma}$ Production in $p p$ Collisions at $\sqrt{s}=8 \mathrm{TeV}$ and limits on anomalous quartic gauge couplings with the ATLAS detector," Physical Review Letters, vol. 115, no. 3, Article ID 031802, 2015.

[36] G. Aad, S. Caron, L. Colasurdo et al., "Measurements of $Z \gamma$ and Z $\gamma \gamma$ production in $p p$ collisions at $\sqrt{s}=8 \mathrm{TeV}$ with the ATLAS detector," Physical Review D, vol. 93, no. 11, Article ID 112002, 2016.

[37] G. Aad and ATLAS Collaboration, "Measurements of $W^{ \pm} Z$ production cross sections in $P p$ collisions at $\sqrt{s}=8$ with the ATLAS detector and limits on anomalous gauge boson selfcouplings," Physical Review D, vol. 93, no. 9, Article ID 092004, 2016.

[38] M. Aaboud and ATLAS Collaboration, "Measurement of $W^{ \pm} W^{ \pm}$vector-boson scattering and limits on anomalous quartic gauge couplings with the ATLAS detector," https://arxiv.org/ abs/1611.02428.

[39] M. Fabbrichesi, M. Pinamonti, A. Tonero, and A. Urbano, "Vector boson scattering at the LHC: a study of the $W W \rightarrow W W$ channels with the Warsaw cut," Physical Review D-Particles, Fields, Gravitation and Cosmology, vol. 93, no. 1, Article ID 015004, 2016.
[40] J. Alwall, R. Frederix, S. Frixione et al., “The automated computation of tree-level and next-to-leading order differential cross sections, and their matching to parton shower simulations," Journal of High Energy Physics, vol. 2014, article 79, 2014.

[41] O. J. Éboli, M. C. Gonzalez-Garcia, and S. M. Lietti, "Bosonic quartic couplings at CERN LHC," Physical Review D, vol. 69, no. 9, Article ID 095005, 2004.

[42] G. Moortgat-Pick, T. Abe, G. Alexander et al., "Polarized positrons and electrons at the linear collider," Physics Reports, vol. 460, no. 4-5, pp. 131-243, 2008. 

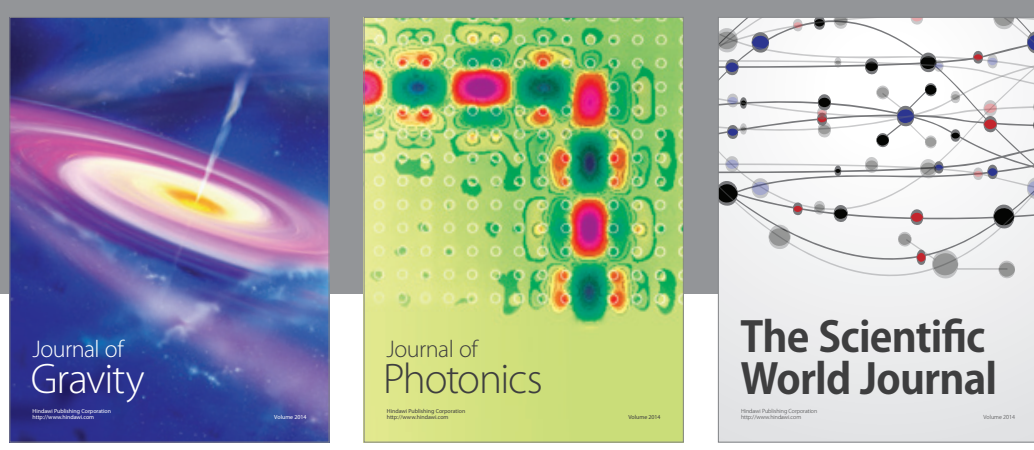

The Scientific World Journal
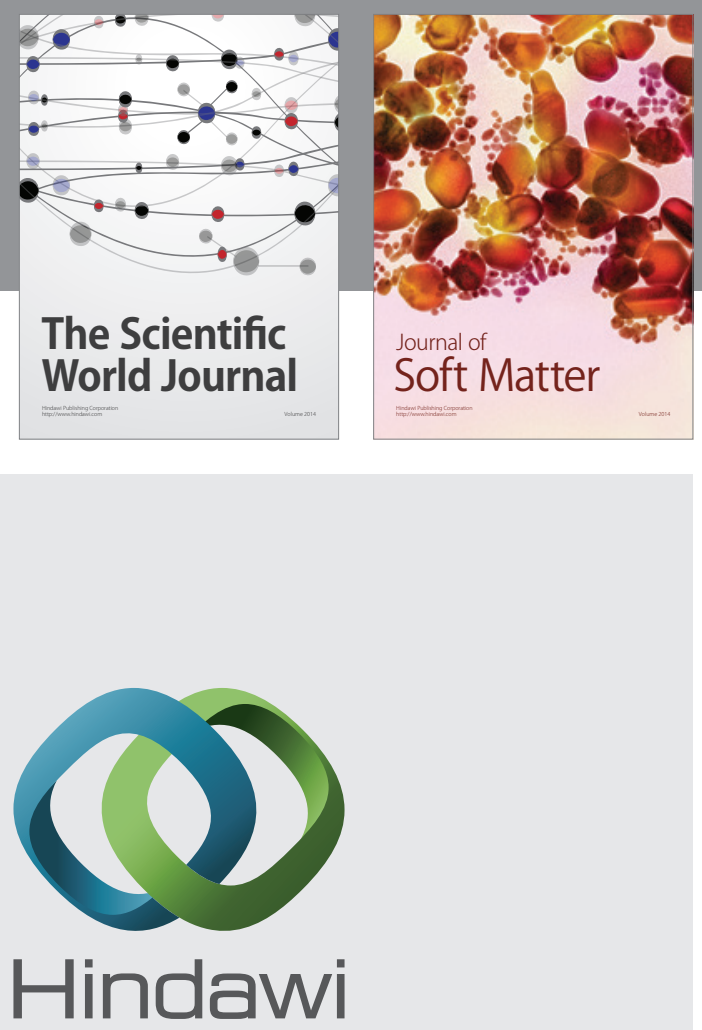

Submit your manuscripts at

https://www.hindawi.com
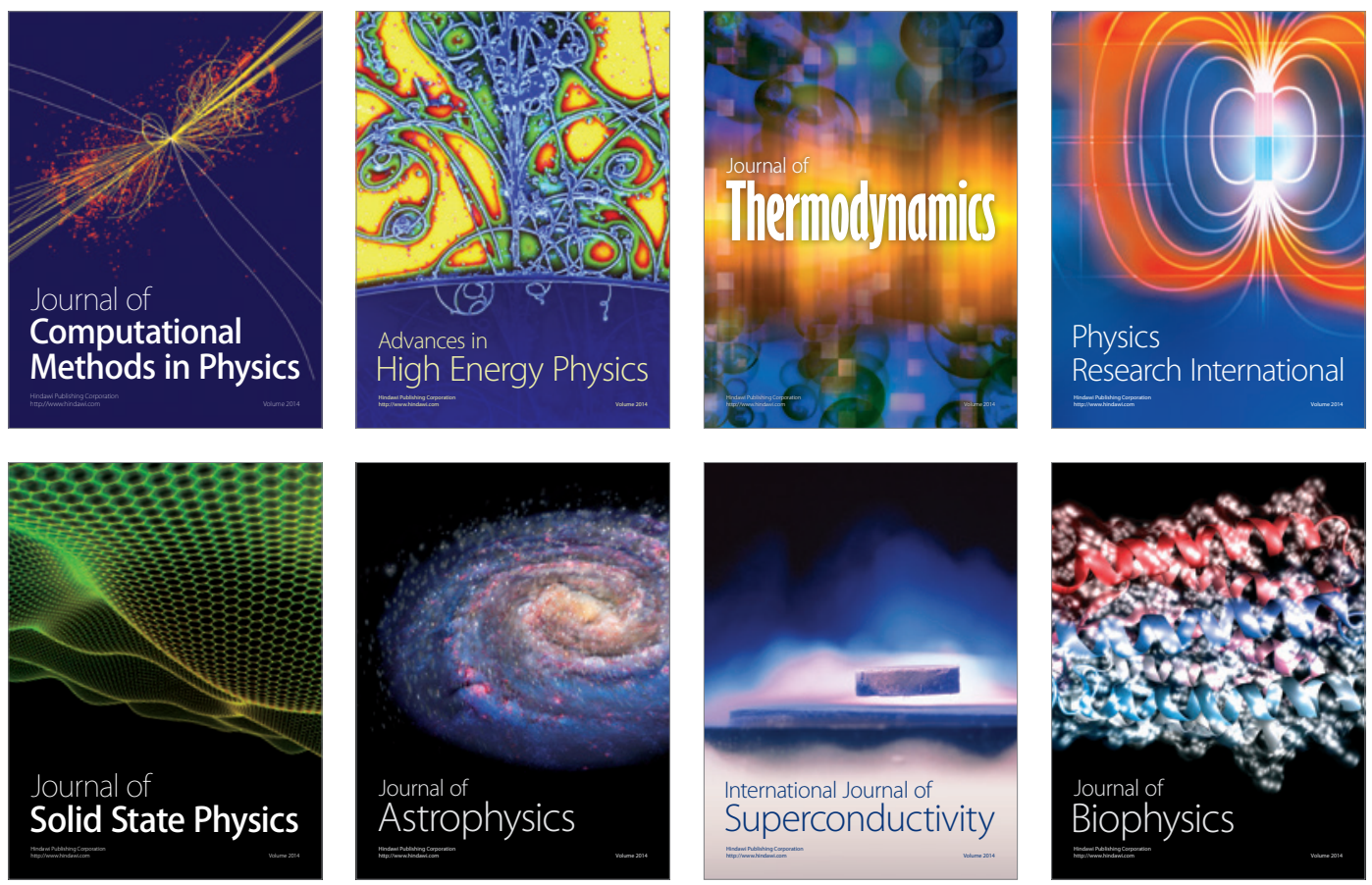
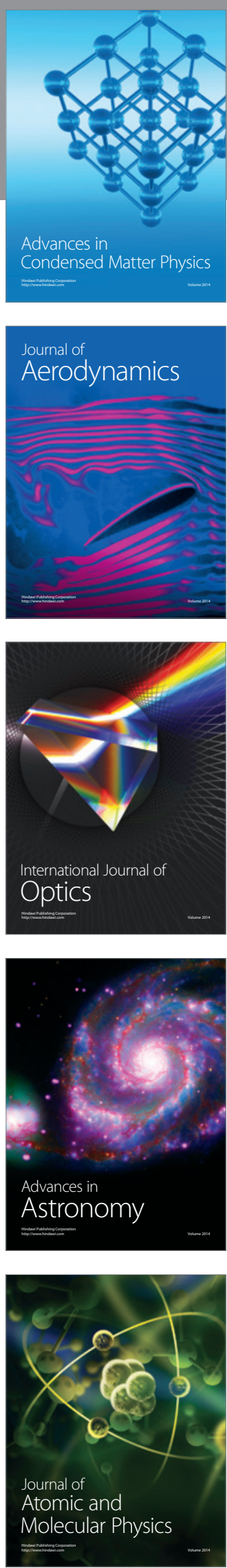